\title{
The Dirichlet problem for degenerate complex Monge-Ampere equations
}

\author{
D.H. Phong And JaCOB Sturm
}

\begin{abstract}
The Dirichlet problem for a Monge-Ampère equation corresponding to a non-negative, possible degenerate cohomology class on a Kähler manifold with boundary is studied. $C^{1, \alpha}$ estimates away from a divisor are obtained, by combining techniques of Blocki, Tsuji, Yau and pluripotential theory. In particular, $C^{1, \alpha}$ geodesic rays in the space of Kähler potentials are constructed for each test configuration.
\end{abstract}

\section{Introduction}

This paper is motivated by two closely related problems: on the one hand, the Dirichlet problem for degenerate complex Monge-Ampère equations on compact Kähler manifolds with boundary, and on the other hand, the existence and regularity of geodesics in the space of Kähler potentials of a given Chern class.

The complex Monge-Ampère equation has been studied extensively over the years. Three particularly influential works have been the work by Yau [38] on non-degenerate equations on compact Kähler manifolds without boundary, by Caffarelli-Kohn-Nirenberg-Spruck [6] on the Dirichlet problem on strongly pseudo-convex domains in $\mathbf{C}^{n}$ (also for non-degenerate equations) and by Bedford-Taylor $[2,3]$ on generalized solutions in the sense of pluripotential theory. Since then, there has been considerable further progress, in particular for $L^{p}$ right-hand sides [20], for existence and regularity in the case of quasi-projective manifolds (see, e.g.,[12, 13, 19, 37]) and for singular canonical metrics and degenerate equations on Kähler manifolds $[14,17,21,30,31,35,36]$. As for the Dirichlet problem, it has been extended to more general domains in $\mathbf{C}^{n}$ [18], and to Kähler manifolds $M$ with boundary $[5,8,26]$ for equations of the form

$$
\left(\Omega+\frac{i}{2} \partial \bar{\partial} \Phi\right)^{m}=F \Omega^{m}, \quad \Omega+\frac{i}{2} \partial \bar{\partial} \Phi \geq 0, \quad \Phi_{\left.\right|_{\partial M}}=\varphi,
$$


when $F \geq 0$ and the given cohomology class $[\Omega]$ is strictly positive definite (and hence is a Kähler form). However, the important case of $[\Omega]$ nonnegative, but possibly degenerate on a Kähler manifold with boundary, has not been fully elucidated.

The degenerate complex Monge-Ampère equation plays an important role in the problem of constant scalar curvature metrics, which is a fundamental problem in Kähler geometry. Let $L \rightarrow X$ be a positive holomorphic line bundle over a compact complex manifold $X$. The positivity of $L$ means that there exists a metric $h_{0}$ on $L$ with positive-definite curvature $\omega_{0} \equiv-\frac{i}{2} \partial \bar{\partial} \log h$. By a well-known conjecture of Yau [39], the existence of such a constant scalar curvature metric $\omega \in c_{1}(L)$ on a compact Kähler manifold $X$ with polarization $L \rightarrow X$ should be equivalent to the stability of $L$ in the sense of geometric invariant theory. Suitable notions of $K$-stability have been proposed since by Tian [34] and Donaldson [16]. But of particular interest to us is yet another notion of stability introduced by Donaldson [15], where the one-parameter subgroups of geometric invariant theory are replaced by geodesic rays in the space $\mathcal{K}$ of Kähler potentials

$$
\mathcal{K}=\left\{\varphi \in C^{\infty}(X) ; \quad \omega_{0}+\frac{i}{2} \partial \bar{\partial} \varphi>0\right\}
$$

equipped with the natural Weil-Petersson metric. The conjecture/question of Donaldson [15] states that the absence of a constant scalar curvature metric should imply the existence of an infinite geodesic ray along which the Mabuchi $K$-energy is decreasing. See e.g. [23, 27] for a survey.

If $(-T, 0] \ni t \rightarrow \varphi(\cdot, t)$ is a path in $\mathcal{K}$, Donaldson [15], Semmes [29] and Mabuchi [22] recognized that the geodesic equation for $\phi$ is equivalent to the following completely degenerate Monge-Ampère equation:

$$
\left(\Omega_{0}+\frac{i}{2} \partial \bar{\partial} \Phi\right)^{m}=0 \quad \text { on } M, \quad \Phi_{|w|=1}=\varphi(z, 0)
$$

where $M=X \times\left\{e^{-T}<|w| \leq 1\right\}, \Omega_{0}$ is the form $\omega_{0}$ pulled back to $M$, and

$$
\Phi(z, w) \equiv \varphi(z, \log |w|), \quad(z, w) \in M .
$$

We refer to $\varphi(\cdot, t)$ (equivalently $\Phi(\cdot, w)$ ) as a geodesic segment when $T$ is finite, and as a geodesic ray when $T$ is infinite. The Monge-Ampère formulation provides a notion of "generalized geodesics": these are rotation invariant, $\Omega_{0}$-plurisubharmonic, generalized solutions $\Phi(z, w)$ of Equation (1.3) in the sense of pluripotential theory. Henceforth, we consider only generalized geodesics, and refer to them just as geodesics for simplicity. 
Dirichlet problem for degenerate complex Monge-Ampere equations147

Using the Monge-Ampère equation, geodesic segments in $\mathcal{K}$ have been constructed by Chen [8] using the method of a priori estimates, and by [24] using pluripotential theory. A detailed analysis of the method of a priori estimates has been provided very recently by Blocki [5]. A partial regularity theory for Equation (1.3) has been provided by [11]. In this paper we are motivated by the stability condition of Donaldson [15] and shall therefore focus on the regularity problem for geodesic rays.

From the point of view of the Monge-Ampère equation, the difference between the segment and the ray cases resides in the behavior of the ray near $w=0$, which is not prescribed by a Dirichlet condition. For geodesic rays associated to a test configuration, two possible approaches have been proposed in $[25,26]$ : the geodesic ray can be modeled on approximating Bergman geodesics, or it can be obtained as a solution of a Monge-Ampère equation on the compactification of the total space of the test configuration, in a given cohomology class $\left[\Omega_{0}\right]$. We shall discuss this in greater detail below. But here we note that [26] treated only the case of $\left[\Omega_{0}\right]$ strictly positive definite. The case where $\left[\Omega_{0}\right]$ is only assumed non-negative is the topic of the present paper.

Our first results concern the general problem of a priori estimates for the Dirichlet problem for the Monge-Ampère equation in the case of possibly degenerate cohomology class $\left[\Omega_{0}\right]$. Our precise set-up is the following.

Let $M$ be a compact complex manifold of complex dimension $m$, with $C^{\infty}$ boundary $\partial M$. Let $\Omega_{0}$ be a smooth, closed, non-negative $(1,1)$-form on $M$,

$$
\Omega_{0} \geq 0,
$$

but not necessarily strictly positive. Assume the following key condition: there exists an effective divisor $E$ on $M$, supported away from the boundary $\partial M$, and an $\varepsilon>0$ such that

$$
\Omega_{0}-\varepsilon[E]>0,
$$

where $[E]$ is the integration current on $E$. We fix such an $\varepsilon>0$ once and for all. If $\mathrm{O}(E)$ is the holomorphic line bundle defined by $E$, and $\sigma(z)$ is the canonical section of $\mathrm{O}(E)$ which vanishes on $E$, condition (1.6) is equivalent to the existence of a metric $H(z)$ on $\mathrm{O}(E)$ satisfying the condition

$$
\Omega_{0}^{\varepsilon} \equiv \Omega_{0}+\frac{i}{2} \partial \bar{\partial} \log H(z)^{\varepsilon}>0 .
$$

Note that the form $\Omega_{0}^{\varepsilon}$ is smooth, so that it defines a Kähler form on $M$. 
Theorem 1.1 (a priori estimates). Let $M, \Omega_{0}$, E be defined as above, and satisfying conditions (1.5) and (1.6). Let $\Phi \in C^{2}(M) \cap C^{4}(M \backslash \partial M)$ be a solution of the Dirichlet problem

$$
\left(\Omega_{0}+\frac{i}{2} \partial \bar{\partial} \Phi\right)^{m}=F\left(\Omega_{0}^{\varepsilon}\right)^{m}, \quad \Omega_{0}+\frac{i}{2} \partial \bar{\partial} \Phi \geq 0, \quad \Phi_{\left.\right|_{\partial M}}=\varphi
$$

where $F>0$ is a smooth function on $M$, and $\varphi \in C^{\infty}(\partial M)$. Let $-B$ be a lower bound for the bisectional curvature of $\Omega_{0}^{\varepsilon}$, with $B \varepsilon>1$ and $b \geq 0$ an upper bound for the scalar curvature. Then we have, with the covariant derivatives and norms written below taken with respect to the metric $\Omega_{0}^{\varepsilon}$ :

(i) $C^{1}$ estimates:

$$
|\nabla \Phi(z)| \leq C_{1}\|\sigma(z)\|^{-\varepsilon A_{1}}, \quad z \in M \backslash E
$$

Here $A_{1}$ is a constant which depends only on an upper bound for $B$ and $\sup _{M}\left|\nabla F^{\frac{1}{m}}\right|$. The constant $C_{1}$ depends on the dimension $m$ and upper bounds for

$$
B, \sup _{M}\left|\nabla F^{\frac{1}{m}}\right|, \sup _{M} F, \sup _{M}|\Phi|, \sup _{M}\|\sigma\|^{2 \varepsilon}, \sup _{\partial M}|\nabla \Phi|, \sup _{\partial M} \frac{1}{\|\sigma\|^{2 \varepsilon}} \text {. }
$$

(ii) $C^{2}$ estimates:

$$
|\Delta \Phi(z)| \leq C_{2}\|\sigma(z)\|^{-\varepsilon A_{2}}, \quad z \in M \backslash E
$$

Here the constant $A_{2}$ depends only on upper bounds for $-\Delta \log F, b$ and $B$. The constant $C_{2}$ depends on the dimension $m$ and upper bounds for

$$
-\Delta \log F, b, B, \operatorname{osc}_{M}(\Phi), \sup _{M}\|\sigma\|^{2 \varepsilon}, \sup _{\partial M}(m+\Delta \phi) .
$$

We note that the constants $C_{1}, C_{2}, A_{1}, A_{2}$ do not depend on either $\inf _{M} F$ or a lower bound for the bisectional curvature of $\Omega_{0}$, an important fact which will be needed for the proofs of Theorems 2 and 3 given below.

Condition (1.6) is a well-known type of condition in the literature. It was introduced by Tsuji [36], who derived the type of $C^{2}$ estimate (ii) in Theorem 1.1 for the Kähler-Ricci flow on manifolds without boundary. Recent related advances are in $[14,17,31,35]$. For manifolds with boundary, $C^{1}$ estimates pose new difficulties. For strictly positive cohomology classes $\left[\Omega_{0}\right]$, the $C^{1}$ estimates have been obtained by Chen [8] and, more recently, by a completely different and more explicit method in the important advance of Blocki [4]. 
For the case of degenerate $\left[\Omega_{0}\right]$ considered here, we adapt the method of Blocki, with a key modification which shows that the maximum principle can be applied at a definite distance from the divisor $E$. We use the a priori estimates in Theorem 1.1, to establish the following theorem:

Theorem 1.2. Let $M, \Omega_{0}, E$ be as above, and satisfy conditions (1.5), (1.6). Consider the Dirichlet problem for the totally degenerate MongeAmpère equation

$$
\left(\Omega_{0}+\frac{i}{2} \partial \bar{\partial} \Phi\right)^{m}=0, \quad \Omega_{0}+\frac{i}{2} \partial \bar{\partial} \Phi \geq 0, \quad \Phi_{\left.\right|_{\partial M}}=0 .
$$

Then the Dirichlet problem (1.11) admits a bounded generalized solution $\Phi$ on the whole of $M$ in the sense of pluripotential theory. Furthermore, for any $0<\alpha<1, \Phi$ is in $C^{\alpha}(M \backslash E)$. If $M$ has a locally flat boundary, in the sense that near each point on $\partial M$, there exists local holomorphic coordinates $\left(z_{1}, \cdots, z_{m}\right)$ so that $\partial M$ is given by $\operatorname{Re} z_{m}=0$, then $\Phi \in C^{1, \alpha}(M \backslash E)$.

We return now to the problem of constructing geodesic rays in the space $\mathcal{K}$ of Kähler potentials in a fixed Chern class $c_{1}(L)$, where $L \rightarrow X$ is a positive line bundle (c.f. (1.2)). This problem has received considerable attention recently, and geodesic rays have been constructed in $[1,8-10,25,26]$ by several different methods.

In particular, in $[25,26]$, it was shown how geodesic rays can be constructed in two different ways starting from a test configuration for $L \rightarrow X$. The open question is how to determine their asymptotic behavior as $t \rightarrow$ $-\infty$. More precisely, a test configuration $\rho$ for $L \rightarrow X$ is an endomorphism

$$
\rho: \mathbf{C}^{\times} \rightarrow \operatorname{Aut}(\mathcal{L} \rightarrow \mathcal{X} \rightarrow \mathbf{C})
$$

with the fibers of $\mathcal{L} \rightarrow \mathcal{X}$ over the point $w=1$ being isomorphic to $L \rightarrow X$ (see [16]). For geodesics, we restrict the fibration to the closed unit disk $\{w \in \mathbf{C} ;|w| \leq 1\}$, with the point $w=0$ corresponding to $t=-\infty$. The fiber $X_{0}$ of $\mathcal{X}$ over $w=0$ is called the central fiber. Let $\mathcal{X}^{\times}$be the complement of $X_{0}$ in $\mathcal{X}$. In [25], the associated geodesic ray is a solution of the complex Monge-Ampère equation on $\mathcal{X}^{\times}$, obtained as a limit of Bergman geodesics, and it was difficult to determine the regularity of the limiting ray. Only for toric varieties has this problem been overcome very recently by Song and Zelditch [32, 33], using a refined semi-classical analysis of norming constants. In [26], the associated geodesic ray was obtained from a priori estimates for a Monge-Ampère equation on the full space $\mathcal{X}$ (more precisely, the full space 
$\tilde{\mathcal{X}}$ of an equivariant resolution,

$$
p: \tilde{\mathcal{X}} \rightarrow \mathcal{X} \rightarrow \mathbf{C}
$$

of $\mathcal{X})$. The ray is then of class $C^{1, \alpha}$ on $\tilde{\mathcal{X}}$ for any $0<\alpha<1$. But the rays constructed in [26] are defined by a strictly positive cohomology class on $\tilde{\mathcal{X}}$ (which is a kind of Dirichlet data at the origin for the punctured disk), while certain more intrinsic cohomology classes exist which are just nonnegative. An important example is the class of the pull-back $p^{*}(\mathcal{L})$. For such classes, a major question is whether the Dirichlet problem for the completely degenerate Monge-Ampère equation can be solved, and whether the solution is $C^{1, \alpha}$ away from the central fiber.

Our next result is that, with Theorems 1.1 and 1.2 , this question can be answered in the affirmative:

Theorem 1.3. Let $L \rightarrow X$ be a positive line bundle over a compact complex manifold $X$, and let $\rho: \mathbf{C}^{\times} \rightarrow \operatorname{Aut}(\mathcal{L} \rightarrow \mathcal{X} \rightarrow \mathbf{C})$ be a test configuration. Fix a metric $h_{0}$ on $L$ with positive curvature $\omega_{0}$. Let $p: \tilde{\mathcal{X}} \rightarrow \mathcal{X} \rightarrow \mathbf{C}$ be an equivariant resolution of $\mathcal{X}$, and let $\tilde{\mathcal{X}}^{\times}=p^{-1}\left(\mathcal{X}^{\times}\right)$be the complement of the central fiber in $\tilde{\mathcal{X}}$. Let $p^{*} \mathcal{L}$ be the pull-back to $\tilde{\mathcal{X}}$ of the line bundle $\mathcal{L}$. Let $H_{0}$ be a metric on $p^{*}\left(\mathcal{L}^{k}\right)$ with non-negative curvature

$$
\Omega_{0} \equiv-\frac{i}{2} \partial \bar{\partial} \log H_{0}
$$

which satisfies the three properties enumerated in Lemma 5.1 below. Then there exists a generalized geodesic ray starting from $h_{0}$, i.e., there exists a bounded, rotation invariant, $\Omega_{0}$-plurisubharmonic function $\Phi$ on $\tilde{\mathcal{X}}$, which is a solution of the following Dirichlet problem:

$$
\left(\Omega_{0}+\frac{i}{2} \partial \bar{\partial} \Phi\right)^{n+1}=0 \quad \text { on } \tilde{\mathcal{X}}, \quad \Phi_{\left.\right|_{\partial \tilde{\mathcal{X}}}}=0
$$

in the sense of pluripotential theory. Furthermore, for any $0<\alpha<1$, the function $\Phi$ is of class $C^{1, \alpha}$ in any compact subset of $\tilde{\mathcal{X}}^{\times}$.

\section{Identities of Blocki, Yau and Aubin}

It is convenient to group together in this section all the identities needed later for the derivation of the a priori $C^{1}$ and $C^{2}$ estimates. Thus consider 
Dirichlet problem for degenerate complex Monge-Ampere equations151

the Monge-Ampère equation

$$
\left(\Omega+\frac{i}{2} \partial \bar{\partial} \Phi\right)^{m}=F \Omega^{m}
$$

on an open manifold $M$, where $\Omega \equiv \frac{i}{2} g_{\bar{k} j} d z^{j} \wedge d \bar{z}^{k}$ is a Kähler form (and in particular positive definite). Let $-B$ be a lower bound for the bisectional curvature of the Kähler form $\Omega$, with $B \geq 0$. Thus $R_{\overline{j i l}{ } a^{i} a^{j}} b^{k} \overline{b^{l}} \geq-B|a|^{2}|b|^{2}$ for all vectors $a^{i}, b^{k}$. All covariant derivatives $\nabla$ in the identities below are taken with respect to the Kähler form $\Omega$. The form $\Omega^{\prime}=\Omega+\frac{i}{2} \partial \bar{\partial} \Phi$ is assumed to be strictly positive. The corresponding metric is denoted by $g_{\bar{k} j}^{\prime}$, and the corresponding covariant derivatives by $\nabla^{\prime}$. Unless otherwise indicated, norms of tensors are taken with respect to $g_{\bar{k} j}$. But there are situations when $\left(g^{\prime}\right)_{\bar{k} j}$ or even a mixture of $g_{\bar{k} j}$ and $\left(g^{\prime}\right)_{\bar{k} j}$ are also used, in which case we shall use sub-indices to indicate which are the relevant metrics. For example, we would write

$$
|\nabla \nabla \Phi|_{\Omega \Omega^{\prime}}^{2}=\left(g^{\prime}\right)^{j \bar{k}} g^{m \bar{p}} \nabla_{j} \nabla_{m} \Phi \nabla_{\bar{k}} \nabla_{\bar{p}} \Phi
$$

and so on.

\subsection{The Blocki identity}

The $C^{1}$ identity is due to Blocki [4]. For a closer analogy with the identities of Yau, Aubin, and Calabi given below, we formulate it in terms of covariant derivatives and in terms of the endomorphism $h=h^{j}{ }_{k}$ defined by

$$
h_{k}^{j}=g^{j \bar{p}} g_{\bar{p} k}^{\prime} .
$$

Let $\gamma: \mathbf{R} \rightarrow \mathbf{R}$ be a smooth real valued function, and let $\Phi$ satisfy the Monge-Ampère equation (2.1). Set

$$
\beta=|\nabla \Phi|_{\Omega}^{2}, \quad \alpha=\log \beta-\gamma(\Phi) .
$$

Then at any interior critical point $p$ of $\alpha$, the following inequality [4] holds:

$$
\begin{aligned}
\Delta^{\prime} \alpha \geq & \frac{1}{\beta}|\bar{\nabla} \nabla \Phi|_{\Omega \Omega^{\prime}}^{2}+\left(\gamma^{\prime}(\Phi)-B-\frac{F_{1}}{\beta^{\frac{1}{2}}}\right) \operatorname{Tr} h^{-1} \\
& +\left(-\gamma^{\prime \prime}(\Phi)+2 \frac{\gamma^{\prime}(\Phi)}{\beta}\right)|\nabla \Phi|_{\Omega^{\prime}}^{2}-(m+2) \gamma^{\prime}(\Phi)-\frac{2}{\beta},
\end{aligned}
$$


where the constant $F_{1}$ is defined by

$$
F_{1}=2 \sup _{M}\left|\nabla\left(F^{\frac{1}{m}}\right)\right|
$$

For the convenience of the reader, we give the derivation of the inequality (2.5), following [5]. First, a direct calculation gives

$$
\begin{aligned}
\Delta^{\prime} \alpha= & \frac{1}{\beta}\left\{|\nabla \nabla \Phi|_{\Omega \Omega^{\prime}}^{2}+|\bar{\nabla} \nabla \Phi|_{\Omega \Omega^{\prime}}^{2}+2 \operatorname{Re}\left(\partial^{p}(\log F) \partial_{p} \Phi\right)\right. \\
& \left.+\left(g^{\prime}\right)^{k \bar{l}} \partial_{p} \Phi R_{k \bar{l}}^{p} \bar{m}_{\bar{m}} \Phi\right\}-\frac{1}{\beta^{2}}|\nabla \beta|_{\Omega^{\prime}}^{2}-\gamma^{\prime \prime}(\Phi)|\nabla \Phi|_{\Omega^{\prime}}^{2}-\gamma^{\prime}(\Phi) \Delta^{\prime} \Phi
\end{aligned}
$$

Here we make use of the identity $\left(g^{\prime}\right)^{p \bar{q}} \nabla_{m} g_{\bar{q} p}^{\prime}=\partial_{m} \log F$, which is obtained by differentiating the Monge-Ampère equation (2.1). It is readily seen that

$$
\begin{aligned}
2\left|\partial^{p}(\log F) \partial_{p} \Phi\right| & \leq 2|\nabla(\log F)| \beta^{\frac{1}{2}} \leq m \frac{F_{1}}{F^{\frac{1}{m}}} \beta^{\frac{1}{2}} \leq\left(\operatorname{Tr} h^{-1}\right) F_{1} \beta^{\frac{1}{2}}, \\
\left(g^{\prime}\right)^{k \bar{l}} \partial_{p} \Phi R_{k \bar{l}}^{p}{ }_{\bar{l}} \partial_{\bar{m}} \Phi & \geq-B \beta \operatorname{Tr} h^{-1} \\
-\Delta^{\prime} \Phi & =-m+\operatorname{Tr} h^{-1},
\end{aligned}
$$

so the only troublesome term is $-\beta^{-2}|\nabla \beta|_{\Omega^{\prime}}^{2}$ (if $\gamma^{\prime},-\gamma^{\prime \prime}$ are chosen to be non-negative, as will be the case later in applications).

The idea in [5] is to cancel this term partially with $|\nabla \nabla \Phi|_{\Omega \Omega^{\prime}}^{2}$. First, introduce the quantity

$$
\Psi_{p} \equiv g^{j \bar{k}} \nabla_{j} \nabla_{p} \Phi \nabla_{\bar{k}} \Phi
$$

On the one hand, we have, by the Cauchy-Schwarz inequality,

$$
\beta|\nabla \nabla \Phi|_{\Omega \Omega^{\prime}}^{2} \geq\left(g^{\prime}\right)^{p \bar{q}} \Psi_{p} \overline{\Psi_{q}},
$$

on the other hand, by a simple calculation

$$
\nabla_{p} \beta=\Psi_{p}+\nabla_{j} \Phi h_{p}^{j}-\nabla_{p} \Phi .
$$

Altogether, we find

$$
\frac{1}{\beta}|\nabla \nabla \Phi|_{\Omega \Omega^{\prime}}^{2} \geq\left|\frac{\nabla \beta}{\beta}-\frac{1}{\beta} \nabla \Phi h+\frac{1}{\beta} \nabla \Phi\right|_{\Omega^{\prime}}^{2} .
$$


Dirichlet problem for degenerate complex Monge-Ampere equations153

So far, all the calculations have been at an arbitrary point. Now, assume that we are at an interior critical point of the function $\alpha$. Then,

$$
\frac{\nabla \beta}{\beta}=\gamma^{\prime}(\Phi) \nabla \Phi
$$

and the preceding expression simplifies (again, with the assumption $\gamma^{\prime}>0$ ) to

$$
\begin{aligned}
\left|\frac{\nabla \beta}{\beta}-\frac{1}{\beta} \nabla \Phi h+\frac{1}{\beta} \nabla \Phi\right|_{\Omega^{\prime}}^{2} & =\left|\gamma^{\prime}(\Phi) \nabla \Phi-\frac{1}{\beta} \nabla \Phi h+\frac{1}{\beta} \nabla \Phi\right|_{\Omega^{\prime}}^{2} \\
& \geq\left(\gamma^{\prime}(\Phi)^{2}\right)|\nabla \Phi|_{\Omega^{\prime}}^{2}-2 \gamma^{\prime}(\Phi)+2 \frac{\gamma^{\prime}(\Phi)}{\beta}|\nabla \Phi|_{\Omega^{\prime}}^{2}-\frac{2}{\beta} .
\end{aligned}
$$

Substituting this inequality in (2.7) gives the desired estimate.

\subsection{Yau and Aubin identities}

The identity of Yau and Aubin is the following, formulated in terms of the endomorphism $h^{j}{ }_{k}$ as in [28],

$$
\begin{aligned}
\Delta^{\prime} \log \operatorname{Tr} h= & \frac{1}{\operatorname{Tr} h}\left(-R+\Delta F+\left(h^{-1}\right)_{m}^{p} R_{p k}^{m}{ }_{k} h_{j}^{k}\right) \\
& +\left\{\left(g^{\prime}\right)^{p \bar{q}} \operatorname{Tr}\left(\nabla_{p}^{\prime} h h^{-1} \nabla_{\bar{q}}^{\prime} h\right)-\frac{\left|\nabla^{\prime} \operatorname{Tr} h\right|^{2}}{(\operatorname{Tr} h)^{2}}\right\} .
\end{aligned}
$$

Again, we provide the derivation. Write

$$
\begin{aligned}
\Delta^{\prime} \operatorname{Tr} h & =\left(g^{\prime}\right)^{p \bar{q}} \partial_{\bar{q}} \partial_{p} \operatorname{Tr} h=\left(g^{\prime}\right)^{p \bar{q}} \operatorname{Tr}\left(\nabla_{\bar{q}}^{\prime}\left[\left(\nabla_{p}^{\prime} h h^{-1}\right) h\right]\right) \\
& =\left(g^{\prime}\right)^{p \bar{q}} \operatorname{Tr}\left(\nabla_{\bar{q}}^{\prime}\left(\nabla_{p}^{\prime} h h^{-1}\right) h\right)+\left(g^{\prime}\right)^{p \bar{q}} \operatorname{Tr}\left(\nabla_{p}^{\prime} h h^{-1} \nabla_{\bar{q}}^{\prime} h\right) .
\end{aligned}
$$

Now the curvatures of $\Omega$ and $\Omega^{\prime}$ are related by the standard formula

$$
\nabla_{\bar{q}}^{\prime}\left(\nabla_{p}^{\prime} h h^{-1}\right)=-(\mathrm{Rm})_{\bar{q} p}\left(\Omega^{\prime}\right)+(\mathrm{Rm})_{\bar{q} p}(\Omega),
$$

where $(\mathrm{Rm})_{\bar{q} p}$ denotes the Riemann curvature tensor viewed as an endomorphism of the tangent bundle. Substituting this identity in the previous 
one gives

$$
\begin{aligned}
\Delta^{\prime} \operatorname{Tr} h= & \left(g^{\prime}\right)^{p \bar{q}} \operatorname{Tr}\left(-\operatorname{Rm}_{\bar{q} p}^{\prime} \cdot h+\operatorname{Rm}_{\bar{q} p} \cdot h\right) \\
& +\left(g^{\prime}\right)^{p \bar{q}} \operatorname{Tr}\left(\nabla_{p}^{\prime} h h^{-1} \nabla_{\bar{q}}^{\prime} h\right) .
\end{aligned}
$$

Thus,

$$
\begin{aligned}
\Delta^{\prime} \log \operatorname{Tr} h= & \frac{\Delta^{\prime} \operatorname{Tr} h}{\operatorname{Tr} h}-\frac{\left|\nabla^{\prime} \operatorname{Tr} h\right|_{\Omega^{\prime}}^{2}}{(\operatorname{Tr} h)^{2}} \\
= & \frac{\left(g^{\prime}\right)^{p \bar{q}} \operatorname{Tr}\left(-R m_{\bar{q} p}^{\prime} \cdot h+R m_{\bar{q} p} \cdot h\right)}{\operatorname{Tr} h} \\
& +\left\{\frac{\left(g^{\prime}\right)^{p \bar{q}} \operatorname{Tr}\left(\nabla_{p}^{\prime} h h^{-1} \nabla_{\bar{q}}^{\prime} h\right)}{\operatorname{Tr} h}-\frac{\left|\nabla^{\prime} \operatorname{Tr} h\right|_{\Omega^{\prime}}^{2}}{(\operatorname{Tr} h)^{2}}\right\}
\end{aligned}
$$

Now $\left(g^{\prime}\right)^{p \bar{q}}=\left(h^{-1}\right)^{p}{ }_{m} g^{m \bar{q}}$, and the middle term on the right-hand side can be rewritten as

$$
\left(g^{\prime}\right)^{p \bar{q}} \operatorname{Tr}\left(\operatorname{Rm}_{\bar{q} p} \cdot h\right)=\left(h^{-1}\right)^{p}{ }_{m} R^{m}{ }_{p}^{\alpha}{ }_{\beta} h^{\beta}{ }_{\alpha} .
$$

As for the first term on the right-hand side, it can be recognized as

$$
\begin{aligned}
\left(g^{\prime}\right)^{p \bar{q}} \operatorname{Tr}\left(\operatorname{Rm}_{\bar{q} p}^{\prime} \cdot h\right) & =\left(\operatorname{Ric}\left(\Omega^{\prime}\right)\right)^{\alpha}{ }_{\beta} h_{\alpha}^{\beta}=\left(g^{\prime}\right)^{\alpha \bar{\gamma}}\left(R^{\prime}\right)_{\bar{\gamma} \beta} h_{\alpha}^{\beta} \\
& =g^{\beta \bar{\gamma}}\left(R^{\prime}\right)_{\bar{\gamma} \beta} .
\end{aligned}
$$

The Monge-Ampère equation implies that

$$
\left(R^{\prime}\right)_{\bar{\gamma} \beta}=R_{\bar{\gamma} \beta}-\partial_{\bar{\gamma}} \partial_{\beta} \log F,
$$

and thus

$$
g^{\beta \bar{\gamma}}\left(R^{\prime}\right)_{\bar{\gamma} \beta}=R-\Delta \log F
$$

Putting this all together, we obtain the desired identity.

Combining Yau's basic inequality

$$
\frac{\left(g^{\prime}\right)^{p \bar{q}} \operatorname{Tr}\left(\nabla_{p}^{\prime} h h^{-1} \nabla_{\bar{q}}^{\prime} h\right)}{\operatorname{Tr} h}-\frac{\left|\nabla^{\prime} \operatorname{Tr} h\right|^{2}}{(\operatorname{Tr} h)^{2}} \geq 0
$$

with the simple identity used earlier $\Delta^{\prime} \Phi=m-\operatorname{Tr} h^{-1}$, and the fact that $(\operatorname{Tr} h)^{-1} \leq \operatorname{Tr} h^{-1}$, we obtain

$$
\Delta^{\prime}(\log \operatorname{Tr} h-A \Phi) \geq \frac{A}{2} \operatorname{Tr} h^{-1}-m A,
$$


Dirichlet problem for degenerate complex Monge-Ampere equations155

for all constants $A$ larger than a constant depending only on lower bounds for $-R$ and $\Delta F$ and the bisectional curvature $R_{\bar{j} j \bar{k} k}$ of the metric $\Omega$.

\section{Proof of Theorem 1}

We can now give the proof of Theorem 1.1. First, define $\Omega_{0}^{\varepsilon}$ by

$$
\Omega_{0}^{\varepsilon}=\Omega_{0}+\frac{i}{2} \partial \bar{\partial} \log H^{\varepsilon}
$$

By hypothesis, the form $\Omega_{0}^{\varepsilon}$ is a Kähler form for some strictly positive $\varepsilon$, which we fix from now on. We use the method of Tsuji [36] and rewrite the original equation (1.8) with $\Omega_{0}^{\varepsilon}$ as background

$$
\left(\Omega_{0}^{\varepsilon}+\frac{i}{2} \partial \bar{\partial} \Phi^{\varepsilon}\right)^{m}=F\left(\Omega_{0}^{\varepsilon}\right)^{m}
$$

where we have defined $\Phi^{\varepsilon}$ by

$$
\Phi^{\varepsilon}(z)=\Phi-\varepsilon \log \|\sigma(z)\|^{2} .
$$

The function $\Phi^{\varepsilon}$ is smooth in $M \backslash E$.

\subsection{Proof of the $C^{1}$ bound}

We now apply Blocki's identity (2.5), with a suitable choice of function $\gamma(x)$. For convenience, we refer to constants satisfying the dependence spelled out in part (i) of Theorem 1 for the constant $C_{1}$ as "admissible constants". Since $\Phi$ is continuous on $M$, the function $\Phi^{\varepsilon}(z)$ is bounded from below. Fix an admissible constant $C$ so that

$$
\inf _{M} \Phi^{\varepsilon} \geq-C+1
$$

Let $-B$, with $B \geq 0$, be a lower bound for the bisectional curvature of the metric $\Omega_{0}^{\varepsilon}$ and assume $B \varepsilon>1$. Set as before $F_{1}^{\varepsilon}=2 \sup \left|\nabla F^{\frac{1}{m}}\right|$. Define the function $\gamma(x)$ to be

$$
\gamma(x)=\left(B+F_{1}^{\varepsilon}+1\right) x-\frac{1}{x+C} .
$$

Note that, unlike in [4], this choice of $\gamma(x)$ does not involve an upper bound for $\Phi^{\varepsilon}$, which is not available in the present case. The function $\gamma(x)$ is smooth 
and bounded from below in the range $[-C+1, \infty), \gamma^{\prime}(x)>B+F_{1}^{\varepsilon}+1$ throughout this range, $\gamma^{\prime \prime}(x)=-2(x+C)^{-3}$, and $\gamma(x) \rightarrow+\infty$ as $x \rightarrow+\infty$.

Consider the function $\alpha$ of (2.4), defined now for equation (3.2), that is, with $\Omega_{0}$ and $\Phi$ replaced now by $\Omega_{0}^{\varepsilon}$ and $\Phi^{\varepsilon}$, respectively,

$$
\beta=\left|\nabla \Phi^{\varepsilon}\right|^{2}, \quad \alpha=\log \beta-\gamma\left(\Phi^{\varepsilon}\right) .
$$

Since $|\Phi|,|\nabla \Phi|$ are all assumed to be continuous on $M$, the function $\alpha$ tends to $-\infty$ near the divisor $E$ (due to the requirement that $B \varepsilon>1$ ). Thus it admits a maximum at a point $p$ which is either on $\partial M$, or in the interior of $M \backslash E$. If $p$ is on $\partial M$, then the desired bound for $|\nabla \Phi|$ follows at once.

Assume then that $p$ is an interior point of $M \backslash E$. It suffices to show that there exists an admissible constant $C_{3}$, so that

$$
\alpha(p) \leq C_{3}
$$

Indeed, this would imply that at an arbitrary point $z \in M \backslash E$, we have

$$
\begin{aligned}
\left|\nabla \Phi^{\varepsilon}(z)\right|^{2} & \leq C_{3} e^{\gamma\left(\Phi^{\varepsilon}(z)\right)} \leq C_{3} e^{\left(B+F_{1}^{\varepsilon}+1\right) \Phi^{\varepsilon}(z)} \\
& \leq C_{4}\|\sigma(z)\|^{-2 \varepsilon\left(B+F_{1}^{\varepsilon}+1\right)}
\end{aligned}
$$

Since $\nabla \Phi^{\varepsilon}=\nabla \Phi+\varepsilon \nabla \log \|\sigma\|^{2}$, we obtain the desired inequality for $|\nabla \Phi|$, with the exponent $A_{1}=2 \varepsilon\left(B+F_{1}+1\right)$, since $B \varepsilon>1$.

We turn to the proof of (3.7). Clearly, we can assume that $\beta>1$ at the point $p$, since otherwise the boundedness from below of $\gamma$ would imply that $\alpha(p)$ satisfies the desired bound. With this assumption, Blocki's estimate (2.5) first simplifies to

$$
\begin{aligned}
\Delta^{\prime} \alpha(p) \geq & \left(\gamma^{\prime}\left(\Phi^{\varepsilon}\right)-B-F_{1}\right) \operatorname{Tr} h^{-1}-\gamma^{\prime \prime}\left(\Phi^{\varepsilon}\right)\left|\nabla \Phi^{\varepsilon}\right|_{\Omega^{\prime}}^{2} \\
& -(m+2) \gamma^{\prime}\left(\Phi^{\varepsilon}\right)-2 .
\end{aligned}
$$

Here we have dropped the manifestly positive terms $\left|\bar{\nabla} \nabla \Phi^{\varepsilon}\right|^{2}, \gamma^{\prime}\left(\Phi^{\varepsilon}\right) \beta^{-1}$ $\left|\nabla \Phi^{\varepsilon}\right|^{2}$. Next, making use of the definition (3.5) of $\gamma$, the inequality simplifies further to

$$
\begin{aligned}
\Delta^{\prime} \alpha(p) \geq & \operatorname{Tr} h^{-1}+\frac{2}{\left(\Phi^{\varepsilon}+C\right)^{3}}\left|\nabla \Phi^{\varepsilon}\right|_{\Omega^{\prime}}^{2} \\
& -(m+2)\left(B+F_{1}^{\varepsilon}+1\right)-(m+4) .
\end{aligned}
$$

Since $p$ is a maximum, $\Delta^{\prime} \alpha(p) \leq 0$. It follows that $\operatorname{Tr} h^{-1}(p) \leq C_{5}$. If $\lambda_{i}$ are the eigenvalues of $g_{\bar{k} j}^{\prime}$ with respect to $g_{\bar{k} j}$ at the point $p$, this implies that all 
Dirichlet problem for degenerate complex Monge-Ampere equations157

$\lambda_{i}^{-1}$ are bounded by admissible constants. On the other hand, $\prod_{j=1}^{m} \lambda_{j}=F$ so $\lambda_{j}$ are also bounded above by admissible constants. In particular, we have

$$
\left|\nabla \Phi^{\varepsilon}(p)\right|_{\Omega}^{2} \leq C_{6}\left|\nabla \Phi^{\varepsilon}(p)\right|_{\Omega^{\prime}}^{2},
$$

where $C_{6}$ is an admissible constant.

The next step is to show that $\|\sigma(p)\|^{-2 \varepsilon}$ is bounded from above by an admissible constant. To see this, we return to the inequality (3.10) and deduce that

$$
\begin{aligned}
\left|\nabla \Phi^{\varepsilon}(p)\right|^{2} & \leq C_{7}\left(\Phi^{\varepsilon}(p)+C\right)^{3} \\
& \leq C_{7}\left(C_{8}+\varepsilon \log \frac{1}{\|\sigma(p)\|^{2}}\right)^{3} .
\end{aligned}
$$

Now, by the definition of admissible constants, we have

$$
\alpha(z) \geq-C_{9}, \quad z \in \partial M
$$

where $C_{9}$ is admissible. On the other hand, $\gamma(x) \geq \frac{1}{C_{10}} x-C_{10}$ with an admissible $C_{10}>0$, so we have

$$
\gamma\left(\Phi^{\varepsilon}(p)\right) \geq \frac{1}{C_{10}} \varepsilon \log \frac{1}{\|\sigma(z)\|^{2}}-C_{11} .
$$

We thus obtain

$$
\begin{aligned}
-C_{9} \leq & \alpha(p) \leq 3 \log \left(C_{8}+\varepsilon \log \frac{1}{\|\sigma(p)\|^{2}}\right) \\
& -\frac{1}{C_{10}} \varepsilon \log \frac{1}{\|\sigma(z)\|^{2}}+C_{12} .
\end{aligned}
$$

This shows that

$$
\varepsilon \log \frac{1}{\|\sigma(p)\|^{2}} \leq C_{13}
$$

and hence $\alpha(p) \leq C_{13}$. This is the desired conclusion, and the proof of the $C^{1}$ estimates is complete.

\subsection{Proof of the $\Delta \Phi$ bound}

For this section, the word "admissible" will now refer to constants with the dependence spelled out for the constant $C_{2}$ in (ii). We consider the expression 
$\operatorname{Tr} h-A \Phi^{\varepsilon}$. Since

$$
\begin{aligned}
\operatorname{Tr} h & =g^{j \bar{k}}\left(g^{\prime}\right)_{\bar{k} j}=m+\Delta \Phi^{\varepsilon}=m+\Delta \Phi-\varepsilon \Delta \log \|\sigma(z)\|^{2} \\
& =m+\Delta \Phi-\varepsilon \Delta \log H,
\end{aligned}
$$

this expression is $C^{2}$ on $M \backslash \partial M$ and extends to a $C^{0}$ function on $M$. On the other hand, $\Phi^{\varepsilon}=\Phi-\varepsilon \log \|\sigma(z)\|^{2} \rightarrow+\infty$ as $z \rightarrow E$. It follows that the expression $\operatorname{Tr} h-A \Phi^{\varepsilon}$ attains its maximum either on the boundary $\partial M$ or an an interior point $p \in M \backslash E$. It suffices to show that

$$
\left(\log \operatorname{Tr} h-A \Phi^{\varepsilon}\right)(p) \leq C_{14}
$$

for some admissible constant $C_{14}$.

By the definition of admissible constants, this inequality holds if $p$ is on $\partial M$. Thus assume that $p$ is in the interior of $M \backslash E$. Since we have then $\Delta^{\prime}(\log \operatorname{Tr} h-A \phi)(p) \leq 0$, it follows from the Yau and Aubin estimate (2.24) that $\operatorname{Tr} h^{-1}(p) \leq C_{15}$. This implies that each eigenvalue $\lambda_{i}$ of $h$ is bounded from below by an admissible constant. Since their product is $F$, each of them is also bounded from above by an admissible constant. For any $z \in M \backslash E$, we can then write

$$
\begin{aligned}
\log \operatorname{Tr} h(z) & \leq A\left(\Phi^{\varepsilon}(z)-\Phi^{\varepsilon}(p)\right)+\log \operatorname{Tr} h(p) \\
& \leq A\left(\Phi^{\varepsilon}(z)-\Phi^{\varepsilon}(p)\right)+C_{16}
\end{aligned}
$$

Thus,

$$
\begin{aligned}
\operatorname{Tr} h(z) & \leq C_{17} e^{A\left(\Phi^{\varepsilon}(z)-\Phi^{\varepsilon}(p)\right)} \\
& =C_{17} e^{A(\Phi(z)-\Phi(p))}\|\sigma(z)\|^{-2 \varepsilon A}\|\sigma(p)\|^{2 \varepsilon A} \\
& \leq C_{18} e^{A \operatorname{osc}(\Phi)}\|\sigma(z)\|^{-2 \varepsilon A} .
\end{aligned}
$$

The proof of the $C^{2}$ estimates is complete.

\section{Proof of Theorem 2}

We wish to solve the Dirichlet problem

$$
\left(\Omega_{0}+\frac{i}{2} \partial \bar{\partial} \Phi\right)^{m}=0, \quad \Omega_{0}+\frac{i}{2} \partial \bar{\partial} \Phi \geq 0, \quad \Phi_{\left.\right|_{\partial M}}=0
$$

where the $(1,1)$-form $\Omega_{0}$ is smooth and non-negative, but may be degenerate. 
Dirichlet problem for degenerate complex Monge-Ampere equations159

\subsection{A viscosity approximation}

As in viscosity methods, we try and obtain a solution to the above generate problem as the limit of a subsequence of solutions of the Dirichlet problem for non-degenerate equations. More specifically, set

$$
\Omega_{s} \equiv \Omega_{0}+s \frac{i}{2} \partial \bar{\partial} \log H^{\varepsilon}, \quad 0 \leq s \leq 1 .
$$

Then $\Omega_{s} \rightarrow \Omega_{0}$ as $s \rightarrow 0$, and for each $0<s \leq 1$, the form $\Omega_{s}$ is a Kähler form. In fact, if $\Omega^{\varepsilon} \equiv \Omega_{0}+\frac{i}{2} \partial \bar{\partial} \log H^{\varepsilon}$ is the Kähler form constructed earlier, we can write

$$
\Omega_{s}=(1-s) \Omega_{0}+s \Omega^{\varepsilon},
$$

which immediately implies that $\Omega_{s}$ is non-degenerate for $0<s \leq 1$.

Consider now for each sufficiently small $s>0$ the following Dirichlet problem for the non-degenerate complex Monge-Ampère equation,

$$
\left(\Omega_{s}+\frac{i}{2} \partial \bar{\partial} \Phi_{s}\right)^{m}=F_{s} \Omega_{s}^{m}, \quad \Omega_{s}+\frac{i}{2} \partial \bar{\partial} \Phi \geq 0,\left.\quad\left(\Phi_{s}\right)\right|_{\partial M}=0,
$$

where the right-hand sides $F_{s}$ are smooth scalar functions on $\bar{M}$, which are defined as follows. Let

$$
\Omega_{s}^{\varepsilon} \equiv \Omega_{s}+\frac{i}{2} \partial \bar{\partial} \log H^{\varepsilon}=\Omega^{\varepsilon}+s \frac{i}{2} \partial \bar{\partial} \log H^{\varepsilon} .
$$

Then $\Omega_{s}^{\varepsilon}>0$ for $s>0$ sufficiently small. Then choose $F_{s}$ so that

$$
\sup _{0<s \leq 1}\left\|F_{s}\right\|_{C^{0}(\bar{M})} \leq 1, \quad \lim _{s \rightarrow 0} \sup _{0<s \leq 1}\left\|F_{s}\right\|_{C^{0}(\bar{M})}=0,
$$

and such that

$$
F_{s}^{(\varepsilon)}=F_{s} \frac{\left(\Omega_{s}\right)^{m}}{\left(\Omega_{s}^{\varepsilon}\right)^{m}}
$$

is a positive constant.

\subsection{Convergence to a generalized solution}

Since $\Omega_{s}$ is a Kähler form for $0<s<1$, and since, by virtue of (4.6), the function $\Phi_{s}=0$ is a subsolution for Equation (4.4), it follows from Theorem 1.3 
of [5] that the Dirichlet problem (4.4) admits a strictly $\Omega_{s}$-plurisubharmonic solution $\Phi_{s}$ which is smooth on $M$.

(a) Uniform $C^{0}$ estimates for $\Phi_{s}$

We claim that there exists a constant $C$ independent of $s$, so that

$$
\left\|\Phi_{s}\right\|_{C^{0}(M)} \leq C
$$

First, by the $\Omega_{s}$-plurisubharmonicity condition, we have

$$
\Delta_{\Omega^{\varepsilon}} \Phi_{s} \geq-\left(\Omega^{\varepsilon}\right)^{j \bar{k}}\left(\Omega_{s}\right)_{\bar{k} j} \geq-C
$$

where $C$ is a constant independent of $s$. If $\hat{\Phi}$ is the solution of the Dirichlet problem

$$
\Delta_{\Omega^{\varepsilon}} \hat{\Phi}=-C, \quad \hat{\Phi}_{\left.\right|_{\partial M}}=0
$$

it follows from the maximum principle for the Laplacian that

$$
\Phi_{s} \leq \hat{\Phi}
$$

for all $0<s \leq 1$. Next, recall that the function 0 is a subsolution of the Dirichlet problem (4.4), in view of the fact that the right-hand side $F_{s}$ satisfies condition (4.6). It follows from the maximum principle for the MongeAmpère equation that

$$
0 \leq \Phi_{s} \quad \text { for all } 0<s \leq 1
$$

The estimate (4.8) follows.

(b) Uniform $C^{1}$ estimates for $\Phi_{s}$ at $\partial M$.

Since $0 \leq \Phi_{s} \leq \hat{\Phi}$, and $\hat{\Phi}$ vanishes at $\partial M$, it follows that the absolute values of the partial derivatives of $\Phi_{s}$ are bounded uniformly, where here, as before, all covariant derviatives and norms are taken with respect to $\Omega_{0}^{\varepsilon}$.

(c) Uniform $C^{1}$ estimates on compact subsets of $M \backslash E$.

We shall show the convergence in $C^{\alpha}$ over $M \backslash E$, of a subsequence of $\Phi_{s}$ to a bounded solution the original equation (4.1) in the sense of pluripotential theory. For this, we shall apply Theorem 1.1 to obtain estimates for the gradient of $\Phi_{s}$ which is independent of $s$. We check the hypotheses of Theorem 1.1 in the present case: First note that Equation (4.1) can be readily 
Dirichlet problem for degenerate complex Monge-Ampere equations161

put in the form of the equations considered in Theorem 1.1

$$
\left(\Omega_{s}+\frac{i}{2} \partial \bar{\partial} \Phi_{s}\right)^{m}=F_{s}^{(\varepsilon)}\left(\Omega^{\varepsilon}\right)^{m}
$$

with the constant function $F_{s}^{(\varepsilon)}$ is defined by (4.7).

Recall that the functions $\Phi_{s}$ are $C^{\infty}$ in $\bar{M}$, with $C^{0}$ norms uniformly bounded in $s$, by (4.8). Applying Theorem 1.1 we deduce the existence of a constants $C, A_{1}>0$, which are independent of $s$ and which satisfy

$$
\left|\nabla \Phi_{s}(z)\right| \leq C\|\sigma(z)\|^{-2 \varepsilon A_{1}} .
$$

This gives the desired uniform $C^{1}$ estimate.

(d) Uniform $C^{1, \alpha}$ estimates on compact subsets of $M \backslash E$ in the case where $M$ has locally flat boundary.

Next we derive the $C^{1, \alpha}$ estimate under the assumption of a locally flat boundary: For non-degenerate Monge-Ampère equations, a priori estimates at the boundary depending on a lower bound for the right-hand side were obtained by Caffarelli et al.[6] and Guan [18] by barrier arguments. As noted by Blocki [5], the same arguments can provide bounds independent of a lower bound for the right-hand side if the boundary is locally flat. The same observation was used implicitly earlier by Chen [8]. The following lemma contains a precise formulation of the a priori estimate that we need:

Lemma 4.1. Let $M$ be a compact complex manifold of dimension $m$, with smooth locally flat boundary, let $U$ be an open neighborhood of $\partial M, \Omega$ a Kähler metric on $U$ and let $F \in C^{\infty}(U)$ a positive smooth function. Suppose $\Phi \in C^{\infty}(U)$ with $\Omega^{\prime}=\Omega+\frac{i}{2} \partial \bar{\partial} \Phi>0$ and $\left(\Omega+\frac{i}{2} \partial \bar{\partial} \Phi\right)^{m}=F \Omega^{m}$. Then there is a constant $C>0$, depending on $m, \Omega$, and upper bounds for $\sup _{U} F$ and $\sup _{U}|\nabla \log F|$, such that

$$
\sup _{\partial M}(m+\Delta \Phi) \leq C \sup _{\partial M}\left(1+|\nabla \Phi|^{2}\right) \cdot \sup _{U}\left(1+|\nabla \Phi|^{2}\right)
$$

where the norms and covariant derivatives are taken with respect to $\Omega$.

For the sake of completeness we provide a self-contained proof, which is a straightforward adaptation of the arguments in Guan [18] and Chen [8].

Proof of Lemma 4.1. We write $\Omega=\frac{i}{2} g_{\bar{\beta} \alpha} d z^{\alpha} \wedge d \bar{z}^{\beta}$ and $\Omega^{\prime}=\frac{i}{2} g_{\bar{\beta} \alpha}^{\prime} d z^{\alpha} \wedge d \bar{z}^{\beta}$. Let $p \in \partial M$ be a point where $\sup _{\partial M}(m+\Delta \Phi)$ is achieved, and choose coordinates $\left(z_{1}, \ldots, z_{m}\right)$ on $U$, centered at $p$ and $\delta=\delta_{g}>0$ so that $g_{\bar{\beta} \alpha}(0)=\delta_{\bar{\beta} \alpha}$ 
and so that

$$
\frac{1}{2} I \leq g(z) \leq 2 I
$$

for all $z$ in the neighborhood $U_{\delta}=\left\{\left(z_{1}, \ldots, z_{m}\right): \sum\left|z_{j}\right|^{2}<\delta, x=\operatorname{Re}\left(z_{m}\right) \geq 0\right\}$.

It suffices to show that for $\alpha<m$ or $\beta<m$, we have

$$
\left|\frac{\partial^{2} \Phi}{\partial z_{\alpha} \partial z_{\bar{\beta}}}(0)\right| \leq C\left(\sup _{\partial M}|\nabla \Phi|+1\right)\left(\sup _{U}|\nabla \Phi|+1\right) .
$$

To see this, first note we may assume $\alpha=m$ or $\beta=m$, for otherwise, the left side of (4.16) vanishes identically since $\Phi$ vanishes on the boundary. Next we observe that

$$
\operatorname{det}\left(\delta_{\bar{\beta} \alpha}+\partial_{\alpha} \partial_{\bar{\beta}} \Phi\right)=F \cdot \operatorname{det}(g)
$$

so

$$
1+\frac{\partial^{2} \Phi}{\partial z_{m} \partial \bar{z}_{m}}(0)=F+\sum_{\alpha=1}^{m-1} \frac{\partial^{2} \Phi}{\partial z_{\alpha} \partial \bar{z}_{m}} \cdot \frac{\partial^{2} \Phi}{\partial z_{m} \partial \bar{z}_{\alpha}}
$$

and thus Lemma 4.1 follows from (4.16).

Now we prove (4.16): we assume $\alpha<m$ and $\beta=m$. Let $D= \pm \frac{\partial}{\partial x_{\alpha}}$ or $\pm \frac{\partial}{\partial y_{\alpha}}$ where $x_{\alpha}=\operatorname{Re}\left(z_{\alpha}\right), y_{\alpha}=\operatorname{Im}\left(z_{\alpha}\right)$ and $\alpha<m$. Let $x=\operatorname{Re}\left(z_{m}\right)$. Then we must show

$$
\left(\frac{\partial}{\partial x} D \Phi\right)(0) \leq C\left(\sup _{\partial M}|\nabla \Phi|+1\right)\left(\sup _{U}|\nabla \Phi|+1\right) .
$$

Suppose we could show that $\theta=C a \Phi-D \Phi \geq 0$ on $U_{\delta}$ with $a=\sup _{U}|\nabla \Phi|$ $+1)$. Since $\theta(0)=0$ it would follow that $\frac{\partial \theta}{\partial x}(0) \geq 0$ which would then yield (4.17).

To show $\theta \geq 0$ on $U_{\delta}$ it suffices to show

(a) $\theta \geq 0$ on $\partial U_{\delta}$,

(b) $\Delta^{\prime} \theta \leq 0$.

Now $\Phi \geq 0$ and $D \Phi=0$ if $x=\operatorname{Re}\left(z_{m}\right)=0$. Thus, to achieve (a), we try to modify $\theta$ as follows: $\theta=C a \Phi+\frac{a}{\delta}|z|^{2}-D \Phi$. Since $D \Phi=0$ when $x=0$, we see that if $D \Phi \neq 0$ on $\partial\left(U_{\delta}\right)$, we must have $|z|^{2}=\delta$. Thus condition (a) holds. Note that we still have $\theta(0)=0$ and we have not changed the value of $\frac{\partial \theta}{\partial x}(0)$. 
Dirichlet problem for degenerate complex Monge-Ampere equations163

Next we check (b):

$$
\Delta^{\prime} \Phi=\left(g^{\prime}\right)^{\alpha \bar{\beta}}\left(g_{\bar{\beta} \alpha}^{\prime}-g_{\bar{\beta} \alpha}\right)=m-\sum_{\alpha=1}^{m} \frac{1}{\lambda_{\alpha}},
$$

where $\lambda_{\alpha}$ are the eigenvalues of $g^{\prime}$ with respect to $g$.

Now $\log \operatorname{det} g^{\prime}=\log F+\log \operatorname{det} g$ implies $\left(g^{\prime}\right)^{\alpha \bar{\beta}}\left(D g_{\bar{\beta} \alpha}+\partial_{\alpha} \partial_{\bar{\beta}} D \Phi\right)=D$ $\log F+D \log \operatorname{det} g$ so

$$
\left|\Delta^{\prime}(D \Phi)\right| \leq K\left(1+\sum \frac{1}{\lambda_{\alpha}}\right) \quad \text { and } \quad \Delta^{\prime}|z|^{2} \leq 2 \sum \frac{1}{\lambda_{\alpha}} .
$$

Here $K$ is a constant depending only on $g$ (more precisely, $D g_{\bar{\beta} \alpha}$ ) and on $\sup _{M}|\nabla \log F|$. Thus

$$
\Delta^{\prime}\left(\left(K+\frac{2}{\delta}\right) a \Phi+\frac{a}{\delta}|z|^{2}-D \Phi\right) \leq C a(m+1) .
$$

We are trying to show that $\Delta^{\prime} \theta \leq 0$ so this is not quite what we wanted. Thus we modify one more time: Let $C=K+\frac{2}{\delta}$ and define

$$
\theta=C a \Phi+\frac{a}{\delta}|z|^{2}+C a\left[x-N x^{2}\right]-D \Phi,
$$

where $N$ is a constant, to be chosen later. Since $x-N x^{2} \geq 0$ if $\delta$ is small enough, we still have $\theta \geq 0$ on $\partial U_{\delta}$. The value $\frac{\partial \theta}{\partial x}(0)$ is replaced by $\frac{\partial \theta}{\partial x}(0)+$ $C_{3} a$, which does not affect the estimate we want. Since $\Delta^{\prime} x^{2}=g^{m, \bar{m}} \geq \frac{1}{\sup \lambda_{\alpha}}$ (the diagonal entries of a hermitian matrix are greater than the smallest eigenvalue of the matrix), we have

$$
\Delta^{\prime} \Phi+\Delta^{\prime}\left(x-N x^{2}\right) \leq m-\sum \frac{1}{\lambda_{\alpha}}-\frac{N}{\sup _{\alpha} \lambda_{\alpha}} \leq m-N^{\frac{1}{m}}\left(\operatorname{det} g^{\prime}\right)^{-1} .
$$

Since $\operatorname{det} g^{\prime}=F \operatorname{det} g$, we have $m-N^{\frac{1}{m}}\left(\operatorname{det} g^{\prime}\right)^{-1}<-3 m$ provided $N$ satisfies the bound: $N^{\frac{1}{m}}>2 m\left(\sup _{M} F\right) 2^{m}$. Now we choose $\delta_{g}$ as before, but also satisfying the condition $\left(\frac{1}{\delta}\right)^{\frac{1}{m}}>2 m\left(\sup _{M} F\right) 2^{n+1}$. Thus,

$$
\begin{aligned}
\Delta^{\prime} \Phi+\Delta^{\prime}\left(x-N x^{2}\right) \leq & -3 m \leq-2(m+1) \text { if }\left(\frac{1}{\delta}\right)^{\frac{1}{n+1}} \\
& >N^{\frac{1}{m}}>2 m\left(\sup _{M} F\right) 2^{m}
\end{aligned}
$$


Now we obtain from (4.20):

$$
\begin{aligned}
\Delta^{\prime} \theta & =\Delta^{\prime}\left(2 C a \Phi+\frac{a}{\delta}|z|^{2}+C a\left[x-N x^{2}\right]-D \Phi\right) \\
& \leq C a(m+1)-2 C a(m+1) \leq 0 .
\end{aligned}
$$

This shows $\theta \geq 0$ and $\theta(0)=0$. Thus,

$$
\frac{\partial}{\partial x}(D \Phi) \leq 2 C a \sup _{\partial M}|\nabla \Phi|+C a \leq 2 C a\left(1+\sup _{\partial M}|\nabla \Phi|\right) .
$$

This establishes (4.17) and the lemma is proved.

We return to the proof of Theorem 1.2. We rewrite Equation (4.13) as follows:

$$
\left(\Omega^{\varepsilon}+\frac{i}{2} \partial \bar{\partial}\left(\Phi_{s}+(s-1) \log \|\sigma\|^{2}\right)\right)^{m}=F_{s}^{(\varepsilon)}\left(\Omega^{\varepsilon}\right)^{m} .
$$

We apply Lemma 4.1 to Equation (4.22). The function $|\nabla \log F|$ in Lemma 4.1 vanishes when $F=F_{s}^{(\varepsilon)}$ and we conclude that, in the flat boundary case, $\left|\Delta \Phi_{s}\right|$ is bounded uniformly on $\partial M$. Thus, in this case we have

$$
\left|\Delta \Phi_{s}(z)\right| \leq C\|\sigma(z)\|^{-2 \varepsilon A_{2}}, \quad z \in M \backslash E,
$$

with $C, A_{1}, A_{2}$ all independent of $s$ and $z$. This completes step (d).

Now choose a subsequence $\Phi_{s_{j}}$ converging in $C^{\alpha}$ (resp. $C^{1, \alpha}$ ) to a function $\Phi$, uniformly on compact subsets of $M \backslash E$. Now Equations (4.4) and (4.6) imply

$$
\int_{M}\left(\Omega_{s}+\frac{i}{2} \partial \bar{\partial} \Phi_{s}\right)^{m}=\int_{M} F_{s} \Omega_{s}^{m} \rightarrow 0 \quad \text { as } s \rightarrow 0 .
$$

Thus the Chern-Levine-Nirenberg inequality [7] implies that the function $\Phi$ satisfies (1.11) and Theorem 2 is proved.

Although it is not stated explicitly in Theorem 1.2, it may be worth noting that the gradient and the $C^{1, \alpha}$ norms (in the case of flat boundary) of the solution $\Phi$ satisfy growth estimates near the divisor $E$ of the form $\|\sigma(z)\|^{-\varepsilon A_{3}}$, where $\varepsilon A_{3}$ is a geometric quantity depending only on $\Omega_{0}$ and $E$.

\section{Test configurations and geodesic rays}

We address now the problem of constructing geodesic rays associated to a given test configuration. 
Dirichlet problem for degenerate complex Monge-Ampere equations165

\subsection{Construction of the degenerate form $\Omega_{0}$}

We adhere to the notation of $\S 1$. Thus $\rho: \mathbf{C}^{\times} \rightarrow \operatorname{Aut}(\mathcal{L} \rightarrow \mathcal{X} \rightarrow \mathbf{C})$ is a test configuration for $L \rightarrow X, h_{0}$ is a metric on $L$ and $\omega_{0}>0$ is its curvature.

Lemma 5.1. There exists a metric $H_{0}$ on $p^{*} \mathcal{L}^{m}$ with curvature $\Omega_{0}$ satisfying the following:

1. $\Omega_{0} \geq 0$ on $\tilde{\mathcal{X}}_{D}$ and $\Omega_{0}>0$ on $\tilde{\mathcal{X}}_{D}^{\times}$.

2. $H_{0}$ and $\Omega_{0}$ are rotation invariant.

3. $\left.\Omega_{0}\right|_{X}=\omega_{0}$.

Proof of Lemma 5.1. The Donaldson imbedding theorem (see Lemma 7 of ([25])) says that for some $k>0$ there is an imbedding

$$
I:\left(\mathcal{L}^{k} \rightarrow \mathcal{X} \rightarrow \mathbf{C}\right) \hookrightarrow\left(O(1) \times \mathbf{C} \rightarrow \mathbf{P}^{N} \rightarrow \mathbf{C}\right) .
$$

Let $H_{1}$ be a rotation invariant metric on $O(1) \times \mathbf{C}$ with positive curvature. For example, we can take $H^{\prime}=h_{F S} e^{-|w|^{2}}$ where $w$ is the parameter in C. Let $H_{2}=(I \circ p)^{*} H_{1}^{1 / k}$, and $\Omega_{2}$ its curvature. Let $\omega_{2}=\left.\Omega_{2}\right|_{X}$. Since $H_{2}$ and $h_{0}$ are two metrics on the same line bundle $L \times D^{\times}$there is a smooth function $\Psi: \tilde{\mathcal{X}}^{\times}=L \times D^{\times} \rightarrow \mathbf{R}$ such that $H_{2}=h_{0} e^{-\Psi(x, w)}$.

Let $\eta: D^{\times} \rightarrow[0,1]$ be a smooth function such that $\eta(w)=1$ if $|w| \leq \frac{1}{3}$ and $\eta(w)=0$ if $|w| \geq 2 / 3$. Let

$$
H_{3}=h_{0} e^{-\eta(w) \Psi(x, w)} .
$$

Then $H_{3}=h_{0}$ if $|w| \geq \frac{2}{3}$ and $H_{3}=H_{2}$ if $|w| \leq \frac{1}{3}$. Moreover, the curvature of $H_{3}$ is positive on the fibers $X \times\{w\}$ for all $w \in D^{\times}$. Let $\alpha>0$ be a large positive number and define $H=H_{3} e^{-\alpha\left(|w|^{2}-1\right)}$. Although $H$ is defined as a metric on $\left.p^{*} \mathcal{L}^{m}\right|_{\tilde{\mathcal{X}}_{D}^{\times}}$it clearly extends to a metric on $\tilde{\mathcal{X}}_{D}^{\times}$which has all the desired properties. This proves Lemma 5.1.

\subsection{Construction of a non-degenerate form $\Omega$}

To apply Theorem 1.2 to the geodesic ray equation, we need a divisor $E$ over $\tilde{\mathcal{X}}_{D}$ with $\Omega_{0}-\varepsilon[E]$ a Kähler class. We construct here such a divisor. 
Lemma 5.2. There exists an effective divisor $E$ supported on the central fiber of $\tilde{\mathcal{X}}_{D}$ and an $\varepsilon>0$ with the following property: $\Omega-\varepsilon[E]$ is a Kähler class (where $[E]$ is the current of integration).

More precisely, we claim that there is a smooth metric $H$ on the line bundle $O(E)$ and a positive Kähler metric $\Omega_{\varepsilon}$ on $\tilde{\mathcal{X}}$, such that

$$
\Omega_{0}-\varepsilon[E]=\Omega_{\varepsilon}-\varepsilon \frac{i}{2} \partial \bar{\partial} \log \|\sigma\|^{2}
$$

where $\sigma$ is the canonical section of $\mathrm{O}(E)$ which vanishes on $E$, and $\|\sigma(z)\|^{2}=$ $|\sigma(z)|^{2} H(z)$.

Proof. In Lemma 2 of [26] it was shown that there is a line bundle $\mathcal{M} \rightarrow \tilde{\mathcal{X}}$ and an integer $k>0$ such that

(1) $p^{*} \mathcal{L}^{m k} \otimes \mathcal{M} \rightarrow \tilde{\mathcal{X}}$ is ample, that it, it has a metric $h_{k}$ of positive curvature.

(2) There is a meromorphic section $\mu: \tilde{\mathcal{X}} \rightarrow \mathcal{M}$ whose retriction to $\tilde{\mathcal{X}}^{\times}$is holomorphic and non-vanishing.

In fact the proof shows that $\mu=\frac{1}{s}$, where $s$ is a global section of $\mathcal{M}^{-1}$ which is non-vanishing outside the central fiber (see the paragraph before (3.8) in $[26])$.

Now let $\Omega_{k}>0$ be the curvature of the metric $h_{k}$ and let $E=\{s=0\}$, so that $\mathcal{M}=O(-E)$. Let $H=h_{k}^{-1} H_{0}^{k}$. Then

$$
-\frac{i}{2} \partial \bar{\partial} \log h=k \Omega_{0}-\Omega_{k}
$$

If we let $\varepsilon=\frac{1}{k}$ and $\Omega_{\varepsilon}=\frac{1}{k} \Omega_{k}$ and $s=\sigma$, then we obtain (5.2).

\subsection{Existence of geodesic rays}

We can now prove Theorem 3: Consider the Dirichlet problem (1.11) for the manifold $M=\tilde{\mathcal{X}}_{D}$ where $\Omega_{0}$ is the non-negative form constructed in Lemma 5.1 , and $\varphi=0$. Letting the divisor $E$ be the divisor constructed in Lemma 5.2 , we readily see that all the hypotheses of Theorem 1.2, part (a) are satisfied. Moreover, the manifold $M$ has a locally flat boundary, so Theorem 1.3 follows from Theorem 1.2. 
Dirichlet problem for degenerate complex Monge-Ampere equations167

\section{Acknowledgments}

The authors would particularly like to thank Jian Song for calling their attention to the work of Tsuji in this context and for some helpful conversations. They would like to thank Zbigniew Blocki for communicating to them his preprint [5] prior to publication, and Steve Zelditch for some stimulating discussions. They would also like to thank Ovidiu Munteanu for providing them with references [13] and [19]. Work supported in part by DMS-07-57372 and DMS-05-14003.

\section{References}

[1] C. Arezzo and G. Tian, Infinite geodesic rays in the space of Kähler potentials, Ann. Sc. Norm. Sup. Pisa (5) 2 (2003), 617-630.

[2] E. Bedford and A. Taylor, The Dirichlet problem for a complex MongeAmpère equation, Invent. Math. 37 (1976), 1-44.

[3] E. Bedford and B.A. Taylor, A new capacity for plurisubharmonic functions, Acta Math. 149 (1982), 1-40.

[4] Z. Blocki, A gradient estimate in the Calabi-Yau theorem, Math. Ann. 344 (2009), 317-327.

[5] Z. Blocki, On the space of Kähler metrics, preprint, 2009.

[6] L. Caffarelli, J.J. Kohn, L. Nirenberg and J. Spruck, The Dirichlet problem for non-linear second-order elliptic equations II. Complex Monge-Ampère equations and uniformly elliptic equations, Commun. Pure Appl. Math. XXXVIII (1985), 209-252.

[7] S.S. Chern, H. Levine and L. Nirenberg, Intrinsic norms on a complex manifold, Global Analysis (Papers in honor of K. Kodaira, University of Tokyo Press, Tokyo) (1969), 119-139.

[8] X.X. Chen, The space of Kähler metrics, J. Differential Geom. 56 (2000), 189-234.

[9] X.X. Chen, Space of Kähler metrics III: on the lower bound of the Calabi energy and geodesic distance, arXiv: math.DG / 0606228.

[10] X.X. Chen and Y. Tang, Test configurations and geodesic rays, arXiv:07074149 [math.DG]. 
[11] X.X. Chen and G. Tian, Geometry of Kähler metrics and foliations by holomorphic discs, Publ. Math. Inst. Hautes Études Sci. No. 107 (2008), $1-107$.

[12] S.Y. Cheng and S.T. Yau, On the existence of a complete Kähler metric on noncompact complex manifolds and the regularity of Fefferman's equation, Comm. Pure Appl. Math. 33(4) (1980), 507-544.

[13] S.Y. Cheng and S.T. Yau, Inequality between Chern numbers of singular Kähler surfaces and characterization of orbit space of discrete group of $S U(2,1)$, Contemporary Math. 49 (1986), 31-44.

[14] J.P. Demailly and N. Pali, Degenerate complex Monge-Ampére equations over compact Kähler manifolds, (2007), arXiv:math.DG/ 0710.5109 .

[15] S.K. Donaldson, Symmetric spaces, Kähler geometry, and Hamiltonian dynamics, Amer. Math. Soc. Transl. 196 (1999), 13-33.

[16] S.K. Donaldson, Scalar curvature and stability of toric varieties, J. Differential Geom. 59 (2002), 289-349.

[17] P. Eyssidieux, V. Guedj and A. Zeriahi, Singular Kähler-Einstein metrics, J. Amer. Math. Soc. 22 (2009), 607-639.

[18] B. Guan, The Dirichlet problem for the complex Monge-Ampère equation and regularity of the pluricomplex Green's function, Comm. Anal. Geom. 6(4) (1998), 687-703.

[19] R. Kobayashi, Kähler-Einstein metric on an open algebraic manifold, Osaka J. Math. 21 (1984), 399-418.

[20] S. Kolodziej, The complex Monge-Ampère equation, Acta Math. 180 (1998), 69-117.

[21] S. Kolodziej and G. Tian, A uniform $L^{\infty}$ estimate for complex MongeAmpère equations, Math. Ann. 342 (2008), 773-787.

[22] T. Mabuchi, Some symplectic geometry on compact Kähler manifolds, Osaka J. Math. 24 (1987), 227-252.

[23] D.H. Phong and J. Sturm, Stability, energy functionals, and KählerEinstein metrics, Comm. Anal. Geom. 11 (2003), 565-597.

[24] D.H. Phong and J. Sturm, The Monge-Ampère operator and geodesics in the space of Kähler potentials, Invent. Math. 166 (2006), 125-149, arXiv: math/0504157. 
Dirichlet problem for degenerate complex Monge-Ampere equations169

[25] D.H. Phong and J. Sturm, Test configurations and geodesics in the space of Kähler potentials, J. Symplectic Geom. 5(2) (2007), 221-247, arXiv: math/0606423.

[26] D.H. Phong and J. Sturm, On the $C^{1,1}$ regularity of geodesics defined by test configurations, arXiv: math/07073956 [math.DG].

[27] D.H. Phong and J. Sturm, Lectures on stability and constant scalar curvature, Current Developments in Mathematics 2007, Int. Press, Somerville, MA, 2009, 101-176.

[28] D.H. Phong, N. Sesum and J. Sturm, Multiplier ideal sheaves and the Kähler-Ricci flow, Comm. Anal. Geom. 15(3) (2007), 613-632, arXiv: math/0611794.

[29] S. Semmes, Complex Monge-Ampere equations and symplectic manifolds, Amer. J. Math. 114 (1992), 495-550.

[30] J. Song and G. Tian, Canonical measures and Kahler-Ricci flow, arXiv: 0802.2570 .

[31] J. Song and B. Weinkove, On the convergence and singularities of the $J$-flow with applications to the Mabuchi energy, Comm. Pure Appl. Math. 61(2) (2008), 210-229.

[32] J. Song and S. Zelditch, Bergman metrics and geodesics in the space of Kähler metrics on toric varieties, arXiv: 0707.3082.

[33] J. Song and S. Zelditch, Test configurations, large deviations and geodesic rays on toric varieties, arXiv: 0712.3599.

[34] G. Tian, Kähler-Einstein metrics with positive scalar curvature, Invent. Math. 130(1) (1997), 1-37.

[35] G. Tian and Z. Zhang, On the Kähler-Ricci flow on projective manifolds of general type, Chinese Ann. Math. Ser. B 27(2) (2006), 179-192.

[36] H. Tsuji, Existence and degeneration of Kähler-Einstein metrics on minimal algebraic varieties of general type, Math. Ann. 281(1) (1988), 123-133.

[37] D. Wu, Kähler-Einstein metrics of negative Ricci curvature on general quasi-projective manifolds, Comm. Anal. Geom. 16(2) (2008), 395-435.

[38] S.T. Yau, On the Ricci curvature of a compact Kähler manifold and the complex Monge-Ampere equation I, Comm. Pure Appl. Math. 31 (1978), 339-411. 
[39] S.T. Yau, Open problems in geometry, Proc. Symposia Pure Math. 54 (1993), 1-28.

Department of Mathematics

Columbia University

NEW YORK

NY 10027

USA

E-mail address: phong@math.columbia.edu

Department of Mathematics

RUTGERS UNIVERSITY

NEWARK

NJ 07102

USA

Received SePtember 22, 2009 